teristics were established from physical studies: large positive gravity anomalies over the circular mare (the mascons), moonquakes both deep and shallow, and widespread magnetisation of the crust. The interpretations of these discoveries are still controversial but we conclude that in its early history the Moon was much more active internally than it is today. If so, why has the Moon died?

The interpretation of gravity field on the near side requires an equally complete survey on the far side. Basins of comparable size to the mare are known to exist on the far side, but they are not filled with lava and whether they have negative or positive gravity anomalies is uncertain. Thus, the exact delineation of gravity above these farside basins is of critical importance to the understanding of the process which resulted in the flooding of mare and the production of mascons on the near side.

Again, the controversy concerning the interpretation of the magnetism of lunar rock centres upon whether the moon generated, between $4 \times 10^{9}$ years and $3.2 \times 10^{9}$ years ago, a general magnetic field which it does not have today. The direction of magnetisation of the lunar crust was impossible to determine by the methods used in terrestrial paleomagnetism; the Apollo samples were unoriented. Instead, interpretation of magnetic anomalies must be used. The existence of an iron core in which a dynamo process might once have been active remains in doubt. A more accurate value of the moment of inertia factor $(0.4$ for a uniform sphere) which is now given as $.392 \pm .003$ could resolve this particular matter.

The definitive solutions of many interesting problems raised by existing data depend on completing the survey of the moon which has been so well begun. To this end the lunar polar orbiter has been conceived in a mission named at present the Terrestrial Bodies Orbiter-Lunar (TBOL). A satellite carrying a variety of remote sensors would be put in lunar orbit about 100 kilometres above the surface so that a complete and relatively detailed sur-

\title{
The experimental programme
}

The geochemical experiments consist of a solid state (germanium) counter for gamma rays and four proportional counters (three looking at the moon and one at the sun) for $X$ rays. The former will detect both the radioactive nuclides $\mathrm{K}, \mathrm{Th}, \mathrm{U}$ and the elements $\mathrm{Fe}, \mathrm{O}, \mathrm{Si}, \mathrm{Ti}, \mathrm{Mg}, \mathrm{Al}$, $\mathrm{Ca}$ (in declining order of detectability) excited by the galactic cosmicray neutrons and by solar flares. The latter will detect the characteristic $X$ rays emitted from $\mathrm{Al}, \mathrm{Si}, \mathrm{Mg}$ for the quiescent sun and also $\mathrm{Ca}, \mathrm{K}, \mathrm{Fe}, \mathrm{Ti}$ during flares. The sampling depth will be $15 \mathrm{~cm}$ for the gamma rays and $10 \mu \mathrm{m}$ for the $\mathrm{X}$ rays and spatial resolutions will be $50 \mathrm{~km}$ and $10 \mathrm{~km}$, respectively. These experiments will provide much more detailed elemental abundance data than has so far been available and will contribute to the understanding of the differentiation of the moon, especially of the highland regions thought to be anorthositic gabbroic in character but only on the basis of fragmentary samples.

Water of the moon, perhaps retained in the regolith of deep craters near the poles, is exciting much interest and the gamma ray experiment may detect it from the $\mathbf{2 . 2 2}$ $\mathrm{MeV}$ line de-excitation of deuterium produced by slow neutrons created in material on the lunar surface by cosmic-ray bombardment.

The radioactivity is known to be greater in the western mare, so that an experiment to detect the surface heat flow is proposed. It may be surprising that this can be done from orbit, but microwave radiometers $(3 \mathrm{~cm}, 10 \mathrm{~cm}, 20 \mathrm{~cm})$-three identical beams with $50 \mathrm{~km}$ resolution-detect the black body radiation from different depths (down to about $5 \mathrm{~m}$ ) in the regolith where, because of its ex- tremely low conductivity, the vertical temperature gradient is large. These radiometers and infrared detectors will also measure the lunar daily inflow and outflow of solar radiation, from which the thermal conductivity of the regolith can be calculated. Thus the distribution of heat sources within the moon will be globally mapped and hypotheses such as convection in the interior will be tested.

Reflectance spectroscopy techniques over wavelengths $0.35-2.5 \mu \mathrm{m}$ will be used to determine the mineralogical composition. This method has already been used effectively to determine the composition of the major satellites of planets. The wavelength of absorption lines from the excitation of electrons from $\mathrm{Fe}^{++}, \mathrm{Fe}^{+++}$and ions of other transition metals are influenced by the crystalline structure of the SiO lattice. It will be supplemented by spectro-stereo images with six filters. The latter instrument, of course, will also provide, in high resolution $(\sim 100 \mathrm{~m})$, further details of the morphology, stratigraphy and, through crater counts, chronology of the lunar surface.

Magnetic maps of the moon will be obtained at the orbital altitude by a 3-component magnetometer accurate to $0.1 \gamma$ and at the surface by an electrostatic analyser of electron fluxes. The latter will detect low energy electrons, spiralling around the lines of the solar wind magnetic field, which are reflected from the convergence of the field lines produced by surface magnetic anomalies. These two methods will complement each other as crustal magnetic anomalies having sharp contrasts of magnetisation look very different at the surface and at heights of $100 \mathrm{~km}$. As various interesting explanations of the magnetisation of lunar rocks have been suggested, some involving cometary and meteoritic impacts, data on the general morphology of magnetic anomalies may establish whether or not these external sources or a magnetic field originating at the lunar core are responsible.

The existence of a lunar core can also be investigated by observing the electromagnetic response of the moon as it enters the fairly steady geomagnetic tail field. The core acts initially as diamagnetic and then the induced dipole moment begins to decay-from this initial decay the free decay time constant of the electric currents in the core can be determined.

The map of the gravity field on the near side will be improved by tracking of the orbiting satellite. For the far side of the moon, complete maps will be made of the gravity field, of which at present nothing is known except by inference from the evolution of the orbital elements of the subsatellites launched in the Apollo 15 and 16 missions. The closely related study of the figure of the moon has been controversial, as the coverage of the laser altimeters on the Apollo 15, 16 and 17 missions has been very limited and the classical earth-bound method, using the geometrical librations which enable height determinations to be made by the parallax principle, were extremely noisy. The radar altimeter in the orbiter should enable the surface to be mapped completely to a high accuracy. The moon departs from a sphere in a variety of ways and the mapping of the moon will be very important to refine our understanding of its non-hydrostatic shape. 